\title{
Recomendaciones -guía- en la lesión aguda medular intraoperatoria en cirugía correctora del raquis
}

\author{
I. Ingelmo Ingelmo ${ }^{\mathrm{a}, 1}$, F. Domínguez Pérez ${ }^{\mathrm{a}, 1}$, J. M. Pinto Corraliza ${ }^{\mathrm{a}, 1}$, N. Fàbregas Juliàa, ${ }^{\mathrm{a}, 2}$ P. Rama-Maceiras ${ }^{\mathrm{a}, 3}$, \\ J. Hernández Palazón ${ }^{\mathrm{a}, 4}$, R. Badenes Quiles ${ }^{\mathrm{a}, 5}$, J. Burgos Flores ${ }^{\mathrm{b}, 1}$, E. Hevia Sierra ${ }^{\mathrm{b}, *, 6}$, H. Mhaidli Hamdan ${ }^{\mathrm{b}, *, 7}$, \\ C. Barrios Pitarque ${ }^{\mathrm{b}, 8}$, G. Pizá Vallespir ${ }^{\mathrm{b}, 9}$, I. Sanpera Trigueros ${ }^{\mathrm{b}, 9}$, P. Doménech Fernández ${ }^{\mathrm{b}, 10}$, I. Álvarez García de Quesada ${ }^{\mathrm{b}, *, 11}$, \\ O. Riquelme García ${ }^{\mathrm{b},{ }^{*}, 12}$, M. García Alonso ${ }^{\mathrm{b}, *, 13}$, R. Ramos Galea ${ }^{\mathrm{b},{ }^{*}, 13}$, P. Gutiérrez Carbonell ${ }^{\mathrm{b}, 10}$, P. Bas Hermida ${ }^{\mathrm{b},{ }^{*}, 14}$, \\ J. Bagó Granell ${ }^{\mathrm{b}, 15}$, I. González Barrios ${ }^{\mathrm{b}, *, 16}$, G. de Blas Beorlegui ${ }^{\mathrm{c}, 1}$, P. Calvo Calleja ${ }^{\mathrm{c}, 17}$, J. Conill Ramónn ${ }^{\mathrm{c}, 15}$, \\ V.E. Cortés Doñate ${ }^{\mathrm{c}, 5}$, M.M. González Hidalgo ${ }^{\mathrm{c}, 18}$, V. Izura Azanza ${ }^{\mathrm{c}, 4}$, M.C. Maeztu Sardiña ${ }^{\mathrm{c}, 19}$, J. Moliner Ibáñez ${ }^{\mathrm{c}, 20}$, \\ I. Regidor Bailly-Bailliere ${ }^{\mathrm{c}, 1}$, A. Sáenz de Cabezón-Álvarez ${ }^{\mathrm{c}, 21}$, C. Santiago Fernández ${ }^{\mathrm{c}, 13}$, S. Soler Algarra ${ }^{\mathrm{c}, 14}$

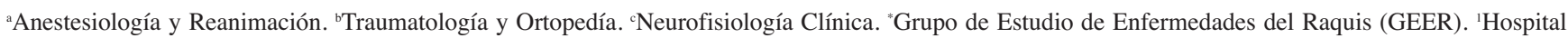 \\ Universitario Ramón y Cajal. Madrid. ${ }^{2}$ Hospital Clínic. Barcelona. ${ }^{3}$ Complejo Hospitalario Universitario. A Coruña. ${ }^{4}$ Hospital Universitario Virgen de la \\ Arrixaca. Murcia. ${ }^{5}$ Hospital Clínico Universitario. Valencia. ${ }^{6}$ Hospital Central Fraternidad Muprespa. Madrid. ${ }^{7}$ Hospital Universitario de Gran Canaria \\ Dr. Negrín. Las Palmas de Gran Canaria. ${ }^{8}$ Universidad de Valencia. ${ }^{9}$ Hospital Son Dureta. Palma de Mallorca. ${ }^{10}$ Hospital General Universitario. Alicante. \\ ${ }^{11}$ Hospital Quirón. Madrid. ${ }^{12}$ Hospital General Universitario Gregorio Marañón. Madrid. ${ }^{13} \mathrm{Hospital}$ Universitario Rio Hortega. Valladolid. ${ }^{14} \mathrm{Hospital}$ La \\ Fe. Valencia. ${ }^{15} \mathrm{Hospital}$ de Traumatología Vall d'Hebron. Barcelona. ${ }^{16} \mathrm{Hospital}$ Reina Sofía. Córdoba. ${ }^{17}$ Complejo Asistencial de Léon. ${ }^{18} \mathrm{Hospital}$ Clínico \\ San Carlos. Madrid. ${ }^{19} \mathrm{Hospital}$ General Universitario Reina Sofía. Murcia. ${ }^{20}$ Hospital Universitario Dr. Peset. Valencia. ${ }^{21}$ Hospital Miguel Servet. \\ Zaragoza.
}

\section{Introducción}

El objetivo del tratamiento quirúrgico para la escoliosis, cifosis y otro tipo de deformidades del raquis es la fusión de las vértebras para que la columna vertebral no pueda curvarse. Se colocan implantes metálicos (barras, tornillos, alambres, placas, etc.), para mantener el raquis mientras las vértebras se fusionan. La artrodesis vertebral se potencia con injerto (autogénico y/o alogénico) de hueso. El abordaje quirúrgico puede ser posterior, anterior o combinación de ambos. La cirugía toracoscópica se puede utilizar en el abordaje anterior del raquis dorsal.

La cirugía correctora del raquis en pediatría y adultos es un procedimiento prolongado (en general más de 4 horas), tiene las complicaciones potenciales de la cirugía mayor (hemorragia grave, hipotermia, infección, embolismo graso o aéreo, hipotensión arterial, etc.) $)^{1,2}$ y entre las patrimoniales, las neurológicas (plejia, paresia) son las más catastróficas ${ }^{3,4}$.

La incidencia de complicaciones neurológicas en la artrodesis raquídea oscila entre $0,26 \%$ a $17 \%$; sin embargo, la mayoría de los estudios se basan en informes retrospectivos de casos no consecutivos. El último estudio de la Scoliosis Research Society refiere una incidencia del 5,7\% de complicaciones mayores intra-

\footnotetext{
Corespondencia:

Dr. D. Ildefonso Ingelmo Ingelmo.

Servicio de Anestesiología y Reanimación.

Hospital Universitario Ramón y Cajal.

Ctra. Colmenar Viejo Km 9,100. 28034 Madrid.

E-mail: iingelmo.hrc@salud.madrid.org
}

Aceptado para su publicación en febrero de 2010 . operatorias en 6.334 niños con escoliosis idiopática 5 . Éstas incluyen dos exitus, uno por hemorragia y otro por embolismo. Hubo 18 pacientes con lesión incompleta medular, de los que 11 se recuperaron completamente.

Hay dos grupos distintos de enfermedades: escoliosis idiopática, que habitualmente presenta buena reserva cardiorrespiratoria y escoliosis secundaria, que puede tener una reserva muy limitada y para quienes el riesgo anestésico es mayor ${ }^{6}$.

La tasa de complicaciones se duplica en los procedimientos combinados anterior más posterior en comparación a ambos por separado. Los pacientes con formas de escoliosis no idiopática presentan mayor riesgo de complicaciones, particularmente hemorrágicas (coagulopatías) y respiratorias, por lo que debe sopesarse el riesgo-beneficio de la corrección quirúrgica ${ }^{7}$. También la mejora pronosticada en la calidad de vida ha de balancearse cuidadosamente frente al riesgo de la cirugía, sobre todo en pacientes con enfermedad neuromuscular progresiva con esperanza de vida limi$\operatorname{tada}^{8,9}$.

La lesión medular más frecuente es la isquémica, causada por la distracción/rotación vertebral o la compresión medular directa por el material de osteosíntesis (barras, tornillos, alambres, etc.). Los déficit sensitivos se producen fundamentalmente por contusión o compresión sobre los cordones posteriores del material de osteosíntesis, mientras que los déficit motores tienen lugar por lesiones isquémicas de la médula espi$\mathrm{nal}^{10,11}$.

La región medular más vulnerable a la isquemia es la antero-lateral (zona por donde desciende la vía piramidal: ipsilateral y contralateral), irrigada por la (fre- 
cuentemente única) arteria espinal anterior. El territorio frontera se encuentra en la unión del tercio posterior (arteria espinal posterior) y el tercio lateral (arteria espinal anterior), donde se localiza el tracto corticoespinal lateral. La circulación anterior suministra a neuronas y sinapsis que son más sensibles a la lesión por hipoperfusión (p.ej. anemia, hipotensión, compresión venosa) que la médula posterior, que además recibe flujo de las interradiculares segmentarias ${ }^{12-14}$.

La prevención de la lesión medular requiere una técnica quirúrgica depurada y una técnica anestésica adecuada para mantener una presión de perfusión medular (PPM) ajustada a las necesidades metabólicas medulares ${ }^{15,16}$.

La monitorización neurofisiológica intraoperatoria (MNI) del sistema nervioso permite detectar las lesiones neurológicas en el momento en que se producen y antes de que se vuelvan irreversibles ${ }^{14,17,18}$; en contraposición a métodos tradicionales que detectan la lesión al finalizar la intervención; como p.ej. la controverti$\mathrm{da}^{4}$ prueba del despertar intraoperatorio (Stagnara wake up test $)^{19} \mathrm{o}$ el test del clonus del tobillo, que actualmente se considera como una prueba no consistente para evaluar la función de la médula espinal ${ }^{20,21}$.

Varios estudios multicéntricos ${ }^{11,22-25}$ han mostrado que la realización de la MNI de la función medular espinal durante la cirugía de la columna ha reducido la incidencia de lesión neurológica postquirúrgica en un $50 \%$, por lo que la MNI es un estándar en la cirugía del raquis ${ }^{14,21}$. Las técnicas quirúrgicas son cada vez más agresivas, lo que permite mayores grados de corrección pero al mismo tiempo han incrementado la posibilidad de déficit postoperatorio ${ }^{4}$.

Actualmente, las metodologías recomendadas ${ }^{26}$ incluyen un registro multimodal de potenciales evocados motores (PEM) $)^{11,22,25,27-29}$ y sensitivos (PESs) ${ }^{30-32}$ para asegurar la integridad de todos los tractos. Aunque en el desarrollo histórico de la monitorización neurofisiológica intraoperatoria el registro de los PESs fue la única herramienta disponible, la aparición de resultados falsos negativos (PESs conservado pero presencia de déficit neurológico motor $)^{33}$ mostró la necesidad de monitorizar las vías motoras, lo cual no fue posible hasta finales de la década de los 90 del siglo pasado por insuficiencia en el desarrollo tecnológico. El PEM obtenido mediante la estimulación eléctrica transcraneal con registro de potenciales musculares es el método indicador más sensible para detectar la isquemia medular ${ }^{26,28,34}$.

En ausencia de variaciones fisiológicas o anestésicas se establece como signo de alarma un descenso $>50 \%$ en la amplitud o un aumento de la latencia $>10 \%$ en el registro de los PESs o una desaparición o reducción significativa de la amplitud del potencial, respecto al basal, de los $\mathrm{PEM}^{4,21,26,35}$. Si los potenciales evocados se pierden de forma completa y persistente, existe un alto riesgo de daño medular; mientras que las pérdidas transitorias durante varios minutos, con recuperación posterior, no conllevan un elevado riesgo de afectación ${ }^{26}$.

Los potenciales evocados deben registrarse de forma continua y de forma especial durante la colocación de la instrumentación y corrección de la deformidad. La monitorización intraoperatoria de los PESs y PEM requiere modificar la técnica anestésica, ya que la mayoría de los fármacos anestésicos deprimen las vías polisinápticas incluidas en la transmisión del impulso nervioso $0^{8,18,36-38}$. Durante el prolongado procedimiento quirúrgico, un registro eficaz puede conseguirse mediante altas dosis de opiáceos, p. ej. remifentanilo 0,3-1 $\mu \mathrm{g} \mathrm{kg}^{-1} \mathrm{~min}^{-1}$ con bajas dosis de propofol y nula o mínima utilización de $\mathrm{N}_{2} \mathrm{O}$ y/o agentes halogenados (< 1 CAM: concentración alveolar mínima). La dexmedetomidina puede utilizarse como adyuvante de la anestesia sin que afecte a la $\mathrm{MNI}^{39-41}$.

Para un registro eficaz de los PEM por estimulación transcraneal, hay que minimizar el empleo de bloqueantes neuromusculares (BNM) o bien realizar su titulación apropiada mediante perfusión continua y monitorización del grado de bloqueo neuromuscular en nervio periférico, permitiendo como mínimo dos respuestas en la secuencia de cuatro estímulos (TOF: "train of four"). La controversia se debe a que una anestesia intravenosa total pura sin BNM es difícil de mantener en procedimientos prolongados debido a: factores metabólicos (en pediatría), disminución de la compliance tóraco-pulmonar (posición prono) e inestabilidad hemodinámica (no permite utilizar planos profundos de hipno-analgesia) $)^{42}$. Por otro lado, cuando se utilizan otros métodos de monitorización como la estimulación medular epidural ${ }^{43-45}$ para obtener el potencial neurogénico, la técnica anestésica debe cambiarse y utilizar un BNM profundo (TOF: 0/4) debido a que las sacudidas musculares que provoca el estímulo pueden ocasionar movimientos súbitos del paciente que dificultan el acto anestésico-quirúrgico ${ }^{31}$.

Otro aspecto a tener en cuenta es la atenuación ("anesthetic fade") de las respuestas motoras tras un tiempo de cirugía, a pesar de unos niveles anestésicos estables, de la ausencia de variaciones fisiológicas significativas o de la presencia de lesiones neurológicas. En estos casos, el umbral de estimulación es cada vez mayor para poder obtener respuestas musculares, tanto en pacientes sin lesión neurológica como en aquellos con una mielopatía previa. Es importante conocer este fenómeno de causa no muy bien conocida, pues debe diferenciarse de un auténtico cambio en los PEM por lesión neural, y ayuda a reducir la incidencia de falsos positivos ${ }^{46}$. 
La corticoterapia farmacológica en la lesión medular aguda tiene resultados estadísticos significativos, pero son clínicamente irrelevantes. La megadosis de corticoides $^{47-50}$ es controvertida ${ }^{51-54}$ y en su utilización debe sopesarse la relación riesgo/beneficio (Tabla 1). No presenta evidencia para considerarla tratamiento estándar ni de guía clínica. Se recomienda como una opción en el tratamiento, en la que los efectos secundarios pueden ser más consistentes que el beneficio clínico $^{4,55}$.

La isquemia medular provoca un aumento de la presión del líquido cefalorraquídeo (LCR) cráneoespinal, posiblemente por elevación en su producción, obstrucción a su libre circulación y disminución en su reabsorción a nivel medular por la compresión venosa vascular; de modo que ocasiona un descenso de la presión de perfusión medular (PPM) ${ }^{13}$. En la reparación de aneurismas aórticos el drenaje externo controlado $\left(10 \mathrm{~mm} \mathrm{Hg}\right.$ o $\left.13 \mathrm{~cm} \mathrm{H}_{2} \mathrm{O}\right)$ de LCR disminuye la incidencia de déficit neurológico postoperatorio, por lo que sería una terapia razonable en situación inminente de isquemia medular durante la cirugía correctora del raquis s $^{13,56-59}$.

A nivel experimental, la hipotermia sistémica inducida tiene propiedades neuroprotectoras frente a la isquemia cerebral ${ }^{60}$ y medular ${ }^{61,62}$, aunque en el neurotrauma y la neurocirugía vascular no ha conseguido mejorar el resultado neurológico ${ }^{63,64}$. En la profilaxis de la isquemia medular durante la cirugía aórtica la hipotermia regional medular mediante enfriamiento epidural demuestra citoprotección ${ }^{65}$, aunque por lo engorroso del procedimiento se opta por la hipotermia sistémica ${ }^{13}$. La evidencia científica también sugiere utilizar la hipotermia terapéutica en pacientes con lesión medular aguda ${ }^{66}$.

El bloqueo neuroaxial mediante anestésicos locales disminuye el consumo metabólico medular $\left(\mathrm{CMRO}_{2 \mathrm{MEDU}}\right)$ al bloquear la transmisión del impulso nervioso, por lo que en situación de isquemia medular podría ser un tratamiento razonable. Sin embargo, al impedir la MNI sería una de las últimas formas de terapia. Por otro lado, la lidocaína por vía intravenosa ha demostrado a nivel experimental disminuir la lesión neurológica en la isquemia medular ${ }^{4}$.

El control efectivo del dolor postoperatorio y la rehabilitación activa requieren un régimen multimodal del manejo del dolor. Opiáceos sistémicos y/o espinales (morfina intratecal: $5 \mu \mathrm{g} \mathrm{kg}^{-1}$ ), anestesia locoregional (epidural, paravertebral) y paracetamol son los más empleados. Los antiinflamatorios no esteroideos (AINE) están controvertidos a causa de sus efectos sobre la fusión ósea y la función plaquetaria. Pueden utilizarse regímenes cortos de AINE cuando los drenajes quirúrgicos han sido retirados ${ }^{6,67-69}$.
TABLA 1

\section{Terapia metilprednisolona}

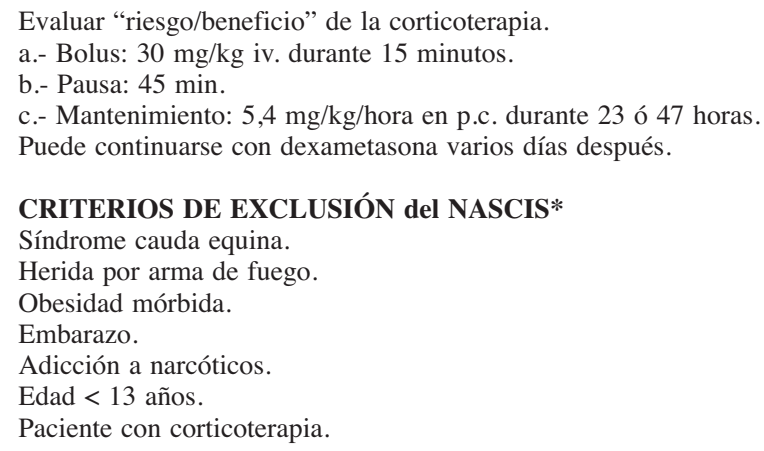

*National Acute Spinal Cord Injury Study ${ }^{48}$.

\section{Recomendaciones de actuación cuando se detecta una situación de riesgo grave de daño medular durante la cirugía correctora de la columna vertebral}

Justificación de las recomendaciones-guía:

- La incidencia de lesiones neurológicas en cirugía de columna vertebral puede alcanzar tasas del 1,2\%, según la enfermedad intervenida. La MNI mediante potenciales evocados y/o electromiografía detecta precozmente la presencia de afectación neurológica en pacientes con anestesia general. La MNI es una técnica con alto valor predictivo (positivo o negativo), por lo que contribuye a reducir la incidencia de lesiones medulares irreversibles.

- La necesidad de MNI durante la cirugía del raquis queda a criterio del equipo quirúrgico, según la alteración por la que se interviene quirúrgicamente. Las personas que participan y quedan implicadas en estas recomendaciones son los médicos especialistas en cirugía de columna vertebral, neurofisiología y anestesiología.

- El respeto y la confianza son los ejes principales que rigen las relaciones entre todos los implicados, especialmente cuando se detecta una situación de alarma.

Cualquier decisión se supone consensuada:

A) El diagnóstico de una situación de riesgo de daño medular potencialmente grave compete al neurofisiólogo en base a la interpretación de cambios en los parámetros objetivos de los potenciales motores y sensitivos que se están registrando de forma periódica.

B) El neurofisiólogo ha de comprobar con rapidez que los cambios en los potenciales evocados registrados no son consecuencia de fallos técnicos ni de una modificación del régimen anestésico o de otros factores externos. 
TABLA 2

\section{Recomendaciones -guía- para la profilaxis de la lesión medular intraoperatoria en cirugía correctora del raquis}

I. Posición quirúrgica adecuada del paciente:

- Evitar compresión vascular (venosa y/o arterial) y nerviosa.

- Facilitar la libre circulación del LCR craneorraquídeo.

II. Medidas generales o de primer nivel neuroprotectoras: Definición de neuroprotección: prevenir o minimizar el daño secundario y terciario.

- Mantener normo o leve hipocapnia y ligera hiperoxemia.

- Mantener normovolemia y euglucemia.

- Mantener $\mathrm{Hb}>8 \mathrm{~g} / \mathrm{dL}$ y ligera hemodilución.

- Mantener normotermia o leve hipotermia.

- Mantener normoosmolalidad o leve hiperosmolalidad sanguínea.

- Mantener normoperfusión medular:

Presión de perfusión medular: PPM = PAM - PLCR

$\mathrm{PPM}>50 \mathrm{~mm} \mathrm{Hg}$ (edad dependiente).

"Transductores (arterial y PLCR) enrasados a "raquis más alto".

III. Medidas específicas o de segundo nivel:

Se evalúa la necesidad o utilidad de medidas específicas de protección medular:

- Aumentar la PPM a 60 ó 70 mm Hg (según edad):

- Elevar la PAM > 80 mm Hg (utilizando inotrópicos

si es necesario).

- Disminuir la $\mathrm{P}_{\mathrm{LCR}}$ mediante drenaje externo controlado (10 mm Hg).

- Utilizar la "megadosis" de metilprednisolona (sopesar riesgo/beneficio).

- Disminuir el consumo metabólico medular $\left(\mathrm{CMRO}_{2 \mathrm{MEDU}}\right)$.

Hb: hemoglogina; LCR = líquido cefalorraquídeo; PAM = presión arterial media; PPM = presión de perfusión medular; PLCR = presión de LCR craneorraquídeo.
C) El anestesiólogo modificará la técnica anestésica y/o tratamiento perianestésico (fármacos anestésicos, hemoglobina, temperatura, presión de perfusión medular, etc.) para optimizar las condiciones de obtención de los potenciales evocados intraoperatorios.

D) El cirujano valorará si ha realizado instrumentaciones o manipulaciones vertebrales o de corrección que puedan haber influido en la modificación o pérdida de los potenciales. En estos supuestos, revertirá las manipulaciones instrumentales recientes en orden inverso, empezando desde la última. Así mismo, debe evaluar si existe algún tipo de exploración quirúrgica o de maniobra coadyuvante que pudiera ayudar al diagnóstico de la afectación de los potenciales o a revertir su efecto.

E) Si transcurridos 10 minutos no se soluciona la situación de alarma de riesgo grave de lesión medular, se aplicará las recomendaciones-guía de lesión aguda medular intraoperatoria (Tabla 2).

F) Si transcurridos 15 minutos más, tampoco se soluciona la situación de alarma de riesgo grave de lesión medular, el cirujano decidirá el conjunto de medidas encaminadas a concluir la intervención qui-

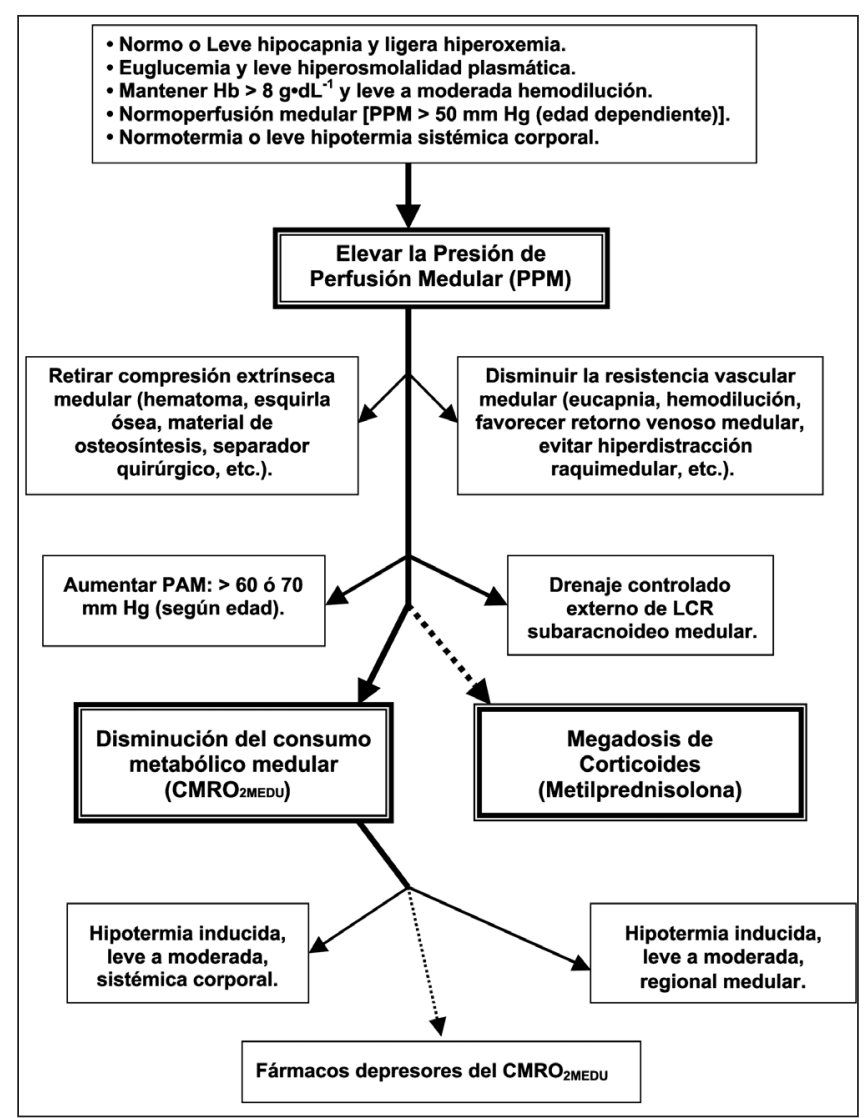

Fig. 1. Algoritmo de actuación para la lesión medular intraoperatoria en cirugía correctora del raquis. "Cuando hay depresión o abolición de potenciales evocados sensitivos y/o motores".

rúrgica. Para reevaluar la integridad medular, el equipo debe ponderar la necesidad de realizar el test de despertar intraoperatorio como opción para valorar un resultado positivo verdadero o falso.

G) Al acabar la intervención, en el mismo quirófano, el equipo realizará una valoración preliminar de la disfunción neurológica medular; antes de trasladar el paciente a la Unidad de Reanimación postquirúrgica o Cuidados intensivos.

En la Figura 1 se resume el algoritmo de actuación recomendado para la lesión medular intraoperatoria durante la cirugía correctora del raquis.

\section{$\overline{\text { BIBLIOGRAFÍA }}$}

1. Carreon LY, Puno RM, Lenke LG, Richards BS, Sucato DJ, Emans JB, et al. Non-neurologic complications following surgery for adolescent idiopathic scoliosis. J Bone Joint Surg Am. 2007;89(11):2427-32.

2. Wills J, Schwend RM, Paterson A, Albin MS. Intraoperative visible bubbling of air may be the first sign of venous air embolism during posterior surgery for scoliosis. Spine (Phila Pa 1976). 2005;30(20):E629-E635.

3. MacEwen GD, Bunnell WP, Sriram K. Acute neurological complications in the treatment of scoliosis. A report of the Scoliosis Research Society. J Bone Joint Surg Am. 1975;57(3):404-8.

4. Pahys JM, Guille JT, D'Andrea LP, Samdani AF, Beck J, Betz RR. 
Neurologic injury in the surgical treatment of idiopathic scoliosis: guidelines for assessment and management. J Am Acad Orthop Surg. 2009;17(7):426-34.

5. Coe JD, Arlet V, Donaldson W, Berven S, Hanson DS, Mudiyam R, et al. Complications in spinal fusion for adolescent idiopathic scoliosis in the new millennium. A report of the Scoliosis Research Society Morbidity and Mortality Committee. Spine (Phila Pa 1976). 2006; 31(3):345-9.

6. Colomina MJ, Godet C. Anestesia para la cirugía de la escoliosis. Estudio preoperatorio y selección de pacientes de riesgo en la cirugía de las deformaciones raquídeas. Rev Esp Anestesiol Reanim. 2005:52(1):24-42.

7. Guigui P, Blamoutier A. Complications of surgical treatment of spinal deformities: a prospective multicentric study of 3311 patients. Rev Chir Orthop Reparatrice Appar Mot. 2005;91(4):314-27.

8. Gambrall MA. Anesthetic implications for surgical correction of scoliosis. AANA J. 2007;75(4):277-85.

9. Perez-Caballero C, Sobrino E, Vazquez JL, Burgos J, Alvarez E, Martos I, et al. Complication of surgery for scoliosis in children with surgically corrected congenital cardiac malformations. Cardiol Young. 2009;19(3):272-7

10. Diab M, Smith AR, Kuklo TR. Neural complications in the surgical treatment of adolescent idiopathic scoliosis. Spine (Phila Pa 1976) 2007;32(24):2759-63.

11. Young PM, Berquist TH, Bancroft LW, Peterson JJ. Complications of spinal instrumentation. Radiographics. 2007;27(3):775-89.

12. Gillilan LA. The arterial blood supply of the human spinal cord. J Comp Neurol. 1958;110(1):75-103.

13. Bicknell CD, Riga CV, Wolfe JH. Prevention of paraplegia during thoracoabdominal aortic aneurysm repair. Eur J Vasc Endovasc Surg. 2009;37(6):654-60.

14. Jameson LC, Sloan TB. Monitoring of the brain and spinal cord. Anesthesiol Clin. 2006;24(4):777-91.

15. Mooney JF, III, Bernstein R, Hennrikus WL, Jr., MacEwen GD. Neurologic risk management in scoliosis surgery. J Pediatr Orthop. 2002;22(5):683-9.

16. Blood pressure management after acute spinal cord injury. Neurosurgery. 2002;50(3 Suppl):S58-S62.

17. Nuwer MR, Daube J, Fischer C, Schramm J, Yingling CD. Neuromonitoring during surgery. Report of an IFCN Committee. Electroencephalogr Clin Neurophysiol. 1993;87(5):263-76.

18. DiCindio S, Schwartz DM. Anesthetic management for pediatric spinal fusion: implications of advances in spinal cord monitoring. Anesthesiol Clin North America. 2005;23(4):765-87.

19. Vauzelle C, Stagnara P, Jouvinroux P. Functional monitoring of spinal cord activity during spinal surgery. Clin Orthop Relat Res. 1973;(93):173-8.

20. Devlin VJ, Schwartz DM. Intraoperative neurophysiologic monitoring during spinal surgery. J Am Acad Orthop Surg. 2007;15(9):549-60.

21. Padberg AM, Wilson-Holden TJ, Lenke LG, Bridwell KH. Somatosensory- and motor-evoked potential monitoring without a wake-up test during idiopathic scoliosis surgery. An accepted standard of care. Spine (Phila Pa 1976). 1998;23(12):1392-400.

22. Eggspuehler A, Sutter MA, Grob D, Jeszenszky D, Dvorak J. Multimodal intraoperative monitoring during surgery of spinal deformities in 217 patients. Eur Spine J. 2007;16 Suppl 2:S188-S196.

23. Nuwer MR, Dawson EG, Carlson LG, Kanim LE, Sherman JE. Somatosensory evoked potential spinal cord monitoring reduces neurologic deficits after scoliosis surgery: results of a large multicenter survey. Electroencephalogr Clin Neurophysiol. 1995;96(1):6-11.

24. Dawson EG, Sherman JE, Kanim LE, Nuwer MR. Spinal cord monitoring. Results of the Scoliosis Research Society and the European Spinal Deformity Society survey. Spine (Phila Pa 1976). 1991;16(8 Suppl):S361-S364

25. Sutter M, Eggspuehler A, Grob D, Jeszenszky D, Benini A, Porchet F, et al. The diagnostic value of multimodal intraoperative monitoring (MIOM) during spine surgery: a prospective study of 1,017 patients. Eur Spine J. 2007;16 Suppl 2:S162-S170.

26. Comisión de la Sociedad Española de Neurofisiología Clínica. Guía práctica para la realización de la monitorización neurofisiológica de la cirugía de la columna. Rev Neurol. 2004;38(9):879-85.

27. Deletis V. Intraoperative neurophysiology of the corticospinal tract of the spinal cord. Suppl Clin Neurophysiol. 2006;59:107-12.
28. Deletis V, Sala F. Intraoperative neurophysiological monitoring of the spinal cord during spinal cord and spine surgery: a review focus on the corticospinal tracts. Clin Neurophysiol. 2008;119(2):24864.

29. Sala F, Lanteri P, Bricolo A. Motor evoked potential monitoring for spinal cord and brain stem surgery. Adv Tech Stand Neurosurg. 2004;29:133-69.

30. Costa P, Bruno A, Bonzanino M, Massaro F, Caruso L, Vincenzo I, et al. Somatosensory- and motor-evoked potential monitoring during spine and spinal cord surgery. Spinal Cord. 2007;45(1):86-91.

31. de Blas-Beorlegui G, Regidor-Bailly-Bailliere I, Fernandez-Lorente J, Leon-Alonso-Cortes J, Montilla-Izquierdo S, Burgos J. Neurophysiological monitoring in spine surgery. Spinal cord stimulation. Rev Neurol. 2004;38(1):68-75.

32. Lopez JR. The use of evoked potentials in intraoperative neurophysiologic monitoring. Phys Med Rehabil Clin N Am. 2004;15(1):63-84.

33. Lesser RP, Raudzens P, Luders H, Nuwer MR, Goldie WD, Morris $\mathrm{HH}$, III, et al. Postoperative neurological deficits may occur despite unchanged intraoperative somatosensory evoked potentials. Ann Neurol. 1986;19(1):22-5.

34. Slimp JC. Electrophysiologic intraoperative monitoring for spine procedures. Phys Med Rehabil Clin N Am. 2004;15(1):85-105.

35. Schwartz DM, Auerbach JD, Dormans JP, Flynn J, Drummond DS, Bowe JA, et al. Neurophysiological detection of impending spinal cord injury during scoliosis surgery. J Bone Joint Surg Am. 2007;89(11):2440-9.

36. Banoub M, Tetzlaff JE, Schubert A. Pharmacologic and physiologic influences affecting sensory evoked potentials: implications for perioperative monitoring. Anesthesiology. 2003;99(3):716-37.

37. Dutton RP. Anesthetic management of spinal cord injury: clinical practice and future initiatives. Int Anesthesiol Clin. 2002;40(3):103-20.

38. Gibson PR. Anaesthesia for correction of scoliosis in children. Anaesth Intensive Care. 2004;32(4):548-59.

39. Tobias JD, Goble TJ, Bates G, Anderson JT, Hoernschemeyer DG. Effects of dexmedetomidine on intraoperative motor and somatosensory evoked potential monitoring during spinal surgery in adolescents. Paediatr Anaesth. 2008;18(11):1082-8.

40. Bala E, Sessler DI, Nair DR, McLain R, Dalton JE, Farag E. Motor and somatosensory evoked potentials are well maintained in patients given dexmedetomidine during spine surgery. Anesthesiology. 2008;109(3):417-25.

41. Anschel DJ, Aherne A, Soto RG, Carrion W, Hoegerl C, Nori P, et al. Successful intraoperative spinal cord monitoring during scoliosis surgery using a total intravenous anesthetic regimen including dexmedetomidine. J Clin Neurophysiol. 2008;25(1):56-61.

42. Pajewski TN, Arlet V, Phillips LH. Current approach on spinal cord monitoring: the point of view of the neurologist, the anesthesiologist and the spine surgeon. Eur Spine J. 2007;16 Suppl 2:S115-S129.

43. Phillips LH, Blanco JS, Sussman MD. Direct spinal stimulation for intraoperative monitoring during scoliosis surgery. Muscle Nerve. 1995;18(3):319-25.

44. Accadbled F, Henry P, de Gauzy JS, Cahuzac JP. Spinal cord monitoring in scoliosis surgery using an epidural electrode. Results of a prospective, consecutive series of 191 cases. Spine (Phila Pa 1976). 2006;31(22):2614-23

45. Wilson-Holden TJ, Padberg AM, Parkinson JD, Bridwell KH, Lenke LG, Bassett GS. A prospective comparison of neurogenic mixed evoked potential stimulation methods: utility of epidural elicitation during posterior spinal surgery. Spine (Phila Pa 1976). 2000;25(18):2364-71.

46. Lyon R, Feiner J, Lieberman JA. Progressive suppression of motor evoked potentials during general anesthesia: the phenomenon of "anesthetic fade". J Neurosurg Anesthesiol. 2005;17(1):13-9.

47. Bracken MB. Methylprednisolone in the management of acute spinal cord injuries. Med J Aust. 1990;153(6):368.

48. Bracken MB, Shepard MJ, Collins WF, Holford TR, Young W, Baskin DS, et al. A randomized, controlled trial of methylprednisolone or naloxone in the treatment of acute spinal-cord injury. Results of the Second National Acute Spinal Cord Injury Study. N Engl J Med. 1990;322(20): 1405-11.

49. Bracken MB, Collins WF, Freeman DF, Shepard MJ, Wagner FW, Silten RM, et al. Efficacy of methylprednisolone in acute spinal cord injury. JAMA. 1984;251(1):45-52. 
50. Bracken MB. The use of methylprednisolone. J Neurosurg. 2000;93(2 Suppl):340-1 .

51. Hugenholtz H, Cass DE, Dvorak MF, Fewer DH, Fox RJ, Izukawa DM, et al. High-dose methylprednisolone for acute closed spinal cord injury--only a treatment option. Can J Neurol Sci. 2002;29(3):227-35.

52. Han YY, Sun WZ. An evidence-based review on the use of corticosteroids in peri-operative and critical care. Acta Anaesthesiol Sin. 2002;40(2):71-9.

53. Hurlbert RJ. The role of steroids in acute spinal cord injury: an evidence-based analysis. Spine. 2001;26(24 Suppl):S39-S46.

54. Hurlbert RJ. Methylprednisolone for acute spinal cord injury: an inappropriate standard of care. J Neurosurg. 2000;93(1 Suppl):1-7.

55. Pharmacological therapy after acute cervical spinal cord injury. Neurosurgery. 2002;50(3 Suppl):S63-S72.

56. Khan SN, Stansby G. Cerebrospinal fluid drainage for thoracic and thoracoabdominal aortic aneurysm surgery. Cochrane Database Syst Rev. 2004;(1):CD003635.

57. Herrera P, Monsma M, Sanchez R, Garrido E, Matoses S, Alepuz R. Tratamiento endovascular de lesiones en aorta torácica con drenaje de líquido cefalorraquídeo. Experiencia inicial en cinco pacientes. Rev Esp Anestesiol Reanim. 2006;53(1):50-3.

58. Kwon BK, Curt A, Belanger LM, Bernardo A, Chan D, Markez JA, et al. Intrathecal pressure monitoring and cerebrospinal fluid drainage in acute spinal cord injury: a prospective randomized trial. J Neurosurg Spine. 2009;10(3):181-93.

59. Fehlings MG. Is cerebrospinal fluid drainage safe and of potential therapeutic benefit after acute traumatic spinal cord injury? J Neurosurg Spine. 2009;10(3):179-80.

60. Busto R, Dietrich WD, Globus MY, Valdes I, Scheinberg P, Ginsberg MD. Small differences in intraischemic brain temperature critically determine the extent of ischemic neuronal injury. J Cereb Blood Flow Metab. 1987;7(6):729-38.

61. Horiuchi T, Kawaguchi M, Kurita N, Inoue S, Nakamura M, Konishi $\mathrm{N}$, et al. The long-term effects of mild to moderate hypothermia on gray and white matter injury after spinal cord ischemia in rats. Anesth Analg. 2009;109(2):559-66.

62. Yu CG, Jimenez O, Marcillo AE, Weider B, Bangerter K, Dietrich WD, et al. Beneficial effects of modest systemic hypothermia on locomotor function and histopathological damage following contusioninduced spinal cord injury in rats. J Neurosurg. 2000;93(1 Suppl):8593.

63. Todd MM, Hindman BJ, Clarke WR, Torner JC. Mild intraoperative hypothermia during surgery for intracranial aneurysm. N Engl J Med. 2005;352(2):135-45.

64. Choi R, Andres RH, Steinberg GK, Guzman R. Intraoperative hypothermia during vascular neurosurgical procedures. Neurosurg Focus. 2009;26(5):E24.

65. Cambria RP, Davison JK. Regional hypothermia for prevention of spinal cord ischemic complications after thoracoabdominal aortic surgery: experience with epidural cooling. Semin Thorac Cardiovasc Surg. 1998;10(1):61-5.

66. Dietrich WD, III. Therapeutic hypothermia for spinal cord injury. Crit Care Med. 2009;37(7 Suppl):S238-S242.

67. Borgeat A, Blumenthal S. Postoperative pain management following scoliosis surgery. Curr Opin Anaesthesiol. 2008;21(3):313-6.

68. Rubio PP, de la Cruz BJ. Unidad para el tratamiento del dolor agudo postoperatorio pediátrico: una experiencia de seis años. Rev Esp Anestesiol Reanim. 2006;53(6):346-53.

69. Rowbotham DJ. COX-2-selective inhibitors: clinical relevance in surgical and acute pain. Eur J Anaesthesiol Suppl. 2002;2511-20. 